\title{
Using the Modified Clavien Grading System to Classify Complications of Percutaneous Nephrolithotomy
}

\author{
Aditya K. Singh Pushpendra K. Shukla Sartaj W. Khan \\ Vazir S. Rathee Udai Shankar Dwivedi Sameer Trivedi \\ Department of Urology, Institute of Medical Sciences, Banaras Hindu University, Varanasi, India
}

\begin{abstract}
Key Words
Percutaneous nephrolithotomy • Complications • Calculus • Clavien
\end{abstract}

\begin{abstract}
Purpose: A modified Clavien classification system has been proposed to grade perioperative complications. We share our experience in grading the complications of percutaneous nephrolithotomy (PNL), according to this new classification. Methods: A total of 809 PNLs performed between 2010 and 2014 were reviewed retrospectively. The modified Clavien classification system, which classifies the perioperative complications into 5 grades, was applied. Grade wise comparison of complications between the patients with simple and complex calculi was done. We also carried out a univariate analysis of different predictors of complications after surgery. Results: A total of 253 perioperative complications were observed in 237 (29.29\%) patients. Most complications were related to bleeding and urinary leakage. Patients with complex calculi had significantly more number of complications across all Clavien groups. In a univariate analysis, positive preoperative urine culture and multiple access for stone clearance were identified to be the independent predictors of complications. Conclusion: The modified Clavien system is a simplistic grading system for classification of postoperative complications. However, it suffers from various shortcomings. Therefore, till the proposition of a more
\end{abstract}

\section{KARGER}

() 2017 S. Karger AG, Basel

Fax +4161306 1234

E-Mail karger@karger.com

www.karger.com
Accessible online at: www.karger.com/cur comprehensive classification system, the modified Clavien system is useful for reporting the complications and short -term outcomes of PNL.

Copyright ๑ 2017 S. Karger AG, Basel

\section{Introduction}

The technique of percutaneous nephrolithotomy (PNL) was first described by Fernström and Johansson in 1976 [1]. Since then, PNL has established itself as a first line treatment for the management of upper urinary tract calculi, especially large and complex renal calculi [2]. The success rate of PNL has been reported to exceed $90 \%$ in modern literature [3]. Perpetual improvisation in the technique and miniaturization of instruments may further lead to better outcomes in terms of increased success rate as well as a reduction in the incidence of complications.

PNL is generally considered a safe and effective procedure. However complications after PNL occur in a significant proportion of patients with some large studies reporting the complications in the range of $15.6-83 \%$ [4, 5]. The literature is replete with large and well designed studies which have reported the complications associated 
Table 1. Descriptive data of the patient cohort

\begin{tabular}{|c|c|}
\hline Variable & Value \\
\hline Age, year $($ mean $\pm S D)$ & $42.7 \pm 14.5(9-78)$ \\
\hline \multicolumn{2}{|l|}{ Sex, $\mathrm{n}$} \\
\hline Male & 456 \\
\hline Female & 353 \\
\hline \multicolumn{2}{|l|}{ Classification of stones, $\mathrm{n}(\%)$} \\
\hline Simple & $147(18.2 \%)$ \\
\hline Comple & $662(81.8 \%)$ \\
\hline Operative time, $\min ($ mean $\pm S D)$ & $57.9 \pm 22.5(20-170)$ \\
\hline Percutaneous access number, n (\%) & $1.6 \pm 0.5(1-5)$ \\
\hline Single access & $598(73.9 \%)$ \\
\hline Multiple access & $211(26.1 \%)$ \\
\hline \multicolumn{2}{|l|}{ Site of Puncture, n (\%) } \\
\hline Supracostal puncture & $129(15.9 \%)$ \\
\hline Subcostal puncture & $680(84.1 \%)$ \\
\hline \multicolumn{2}{|l|}{ Laterality, n (\%) } \\
\hline Unilateral PNL & $745(92.1 \%)$ \\
\hline Bilateral PNL & $64(7.9 \%)$ \\
\hline \multicolumn{2}{|c|}{ Percutaneous nephrostomy tube placement, n (\%) } \\
\hline Yes & $494(61.1 \%)$ \\
\hline No & $315(38.9 \%)$ \\
\hline \multicolumn{2}{|l|}{ Additional Procedures, n (\%) } \\
\hline Endopyelotomy & $42(5.2 \%)$ \\
\hline Ipsilateral URSL & $22(2.7 \%)$ \\
\hline Infundibulotomy & $4(0.4 \%)$ \\
\hline
\end{tabular}

URSL $=$ Ureteroscope lithotripsy.

with PNL accurately. But the lack of standard stratification of complications based on their severity hampers comparison as well as reproducibility of outcome data for quality assessment.

The modified Clavien-Dindo system is a validated and rational classification system which has been used by urologists worldwide to report perioperative complications of various surgeries like laparoscopic radical prostatectomy and laparoscopic donor nephrectomy [6-8]. Recently there has been a drive to apply this system to report perioperative complications of PNL because of its simplistic and comprehensive nature $[9,10]$.

In this article, we retrospectively reviewed our experience with PNL to grade the perioperative complications according to the modified Clavien classification and assess its usefulness and shortcomings in the grading of these complications.

\section{Materials and Methods}

We retrospectively analyzed the data of 809 patients from our PNL registry who underwent PNL between 2010 and 2014. Routine preoperative investigations included complete blood count,
Table 2. Postoperative complications classified by the modified Clavien grading system

\begin{tabular}{lc}
\hline Grade of complications & No. of complications \\
\hline Grade 1 & $37(4.5 \%)$ \\
Fever $\left(>38^{\circ} \mathrm{C}\right)$ & $25(3.1 \%)$ \\
Transient elevation of SCr $(>0.5 \mathrm{mg} / \mathrm{dl})$ & $12(1.4 \%)$ \\
Grade 2 & $128(15.8 \%)$ \\
Blood transfusion & $65(8.0 \%)$ \\
Urine leakage $<24$ hrs & $35(4.3 \%)$ \\
Infections requiring additional antibiotics & \\
$\quad$ Wound infection & $7(0.9 \%)$ \\
$\quad$ Urinary tract infection & $19(2.3 \%)$ \\
Pneumonia & $2(0.2 \%)$ \\
Grade 3a & $52(6.4 \%)$ \\
Renal hemorrhage requiring angioembolization & $4(0.5 \%)$ \\
Postoperative DJ stent placement for urine & $29(3.5 \%)$ \\
leakage & \\
Hemo/pneumothorax requiring chest tube & $11(1.1 \%)$ \\
insertion & \\
Retention due to blood clots & $8(0.9 \%)$ \\
Grade 3b & $25(3.1 \%)$ \\
Ureteric calculus & $8(0.9 \%)$ \\
Collecting system perforation & $5(0.6 \%)$ \\
Infundibular stricture & $1(0.1 \%)$ \\
Urethral stricture & $7(0.8 \%)$ \\
Retained PCN tube requiring removal & $2(0.2 \%)$ \\
Perinephric abscess & $2(0.2 \%)$ \\
Grade 4a & $8(1.0 \%)$ \\
Neighboring organ injury & $1(0.1 \%)$ \\
Myocardial infarction & $3(0.4 \%)$ \\
Acute renal failure & $4(0.5 \%)$ \\
Grade 4b & \\
Sepsis & $2(0.2 \%)$ \\
Grade 5 & $1(0.1 \%)$ \\
Death & \\
& \\
& \\
& \\
&
\end{tabular}

renal function profile, coagulation profile, urinalysis and urine culture. All documented urinary tract infections were treated with appropriate antibiotics before the surgery. A sterile urine culture was ensured before surgery. In patients in whom urine sterilization was not possible prior to surgery, the procedure was done under antibiotic cover. In patients with frank pus on initial puncture, the procedure was deferred, a percutaneous nephrostomy tube inserted and culture sensitive antibiotics were administered.

Baseline imaging in the form of ultrasound abdomen followed by either intravenous urography or CT urography was done for anatomic and functional evaluation of the pelvicalyceal system and morphodensitometric characteristics of the calculi. For the purpose of the study the stones were broadly classified as simple (isolated renal pelvic or calyceal stones) or complex stones (partial or complete staghorn or concomitant renal pelvic and calyceal calculi) [11]. Other parameters like age, sex, laterality, mean operative time, site of puncture, number of punctures and ancillary procedures required were noted in all cases.

PNL was performed in prone position in all patients after placing a ureteral catheter. Puncture was done by bull's eye technique under fluoroscopic guidance. Tract dilatation was done using rigid telescoping coaxial dilators. Tract sizes ranged from 26 to $32 \mathrm{Fr}$. 
Table 3. Comparison of complications between staghorn stone patients and others as stratified by the modified Clavien system

\begin{tabular}{|c|c|c|c|c|c|c|}
\hline Grade & Total $(\mathrm{n}=807)$ & Complex stones $(\mathrm{n}=147)$ & Simple stones $(n=662)$ & $\mathrm{p}$ & Odds ratio & CI \\
\hline 1 & $37(4.5 \%)$ & $17(11.5 \%)$ & $20(3.0 \%)$ & $<0.001$ & 4.19 & $(2.14,8.12)$ \\
\hline 2 & $128(15.8 \%)$ & $57(38.7 \%)$ & $71(10.7 \%)$ & $<0.001$ & 5.2 & $(3.48,7.96)$ \\
\hline $3 b$ & $25(3.1 \%)$ & $11(7.4 \%)$ & $14(2.1 \%)$ & 0.002 & 3.7 & $(1.66,8.42)$ \\
\hline $4 a$ & $8(1.0 \%)$ & $4(2.7 \%)$ & $4(0.6 \%)$ & 0.24 & 4.2 & $(1.13,18.61)$ \\
\hline $4 b$ & $2(0.2 \%)$ & $2(0.1 \%)$ & 0 & & & \\
\hline
\end{tabular}

Table 4. Univariate analysis of predictors of complications

\begin{tabular}{lrrrl}
\hline Variable & $\mathrm{n}$ & \multicolumn{2}{c}{ Complications } & $\mathrm{p}$ \\
\cline { 3 - 4 } & & Yes & No & \\
\hline Gender (Male) & 456 & 131 & 325 & 0.68 \\
Pediatric age & 102 & 27 & 75 & 0.5 \\
Hypertension & 164 & 40 & 124 & 0.12 \\
Diabetes & 78 & 21 & 57 & 0.62 \\
Obesity (BMI > 30) & 48 & 11 & 37 & 0.31 \\
Multiple access tracts & 211 & 74 & 137 & 0.03 \\
Supracostal puncture & 129 & 41 & 88 & 0.28 \\
Operative time (> 120 min) & 97 & 26 & 71 & 0.56 \\
Positive urine culture & 98 & 14 & 84 & 0.02 \\
PCN tube & 494 & 141 & 353 & 0.57 \\
\hline
\end{tabular}

Postoperatively complete blood count and renal profile were obtained. All patients were followed-up 1 month postoperatively. Success was defined as the absence of calculi or the presence of clinically insignificant residual fragments (CIRF) on non-contrast CT scan. CIRFs are those residual calculi which are $<4 \mathrm{~mm}$ in size, asymptomatic, non obstructive and non infectious [12].

Perioperative complications were classified based on the modified Clavien grading system [13]. Comparison between complications arising from simple and complex calculi was made by using the Chi-square and Fisher exact test. A univariate analysis was performed in which the relationship between some independent variables and occurrence of complications was analyzed. Data were expressed as mean plus standard deviation and a p value $<$ 0.05 was considered statistically significant. Odds ratio was calculated and statistical determinations were within the $95 \%$ confidence interval.

\section{Results}

The descriptive data of the patients is summarized in table 1. Overall, PCN tube placement was done in $61.1 \%$ $(\mathrm{n}=494)$ cases, $33.7 \%(\mathrm{n}=273)$ were tubeless (only DJ stent) and $5.2 \%(\mathrm{n}=42)$ were totally tubeless (no DJ stent or PNL). At the 1-month follow-up, an overall suc-

Using the Modified Clavien Grading

System to Classify Complications of

Percutaneous Nephrolithotomy cess rate of $93.2 \%(\mathrm{n}=754)$, including CIRFs observed in $12.1 \%(\mathrm{n}=97)$, was achieved.

Table 2 lists postoperative complications classified by the modified Clavien grading system. Table 3 shows the comparison of complications between patients with simple and complex calculi. Patients with complex calculi showed a statistically significant increased complication rate in modified Clavien categories 1, 2 and 3. Grade 4b and 5 complications were exclusive to the patient cohort with complex calculi. Interestingly, the difference in the incidence of complications was not statistically significant when both these groups were compared with regard to Clavien grade 4a complication group.

Table 4 depicts a univariate analysis of various predictors of complications after PNL. Only multiple accesses and a positive preoperative urine culture appear to correlate with the increased rate of complications after PNL.

\section{Discussion}

Since its introduction in 1976, PNL has established itself as a safe and effective procedure in the management of large renal calculi $[1,2]$. Both these attributes are a result of proper patient selection, continuing technical advances and increasing familiarity with this procedure among practicing urologists. As with all surgical procedures, occurrence of complications is inherent to this procedure as well. Earlier publications reported the complication rates of PNL in the range of 75\% [13]. However, current literature indicates that the complication rate has declined significantly, with most series reporting complication rates of around 30\% [14]. In our study, this rate is $29.29 \%$ which is in agreement with the current literature.

However, there is a significant discrepancy in the available data which arises from the absence of existence of a uniform reporting system. Terms such as major and 
minor have been used but these are not standardized making comparison of complications difficult. A lack of consensus in classifying and inhomogeneity in reporting further adds to the handicap. To address this issue, Clavien et al. [6] in 1992, proposed the general principles to classify complications of surgery and also validated their classification in a survey consisting of 6,336 patients.

Infection is the most common complication that occurs after PNL [15]. It may range in severity from mild postoperative fever to septicemia which may further lead to significant morbidity and even death. The second Clinical Research Office of the Endourological Society study group investigated the incidence of urinary tract infections, post-procedural fever and various factors responsible for it across 96 centers [16]. They reported that 39.8\% patients developed fever during the first 24 hours after the surgery. This rate dropped to about $13 \%$ when the patients were reassessed beyond 24 hours of the procedure. In our study, fever was seen in only $3.1 \%$ of the cases. The lower incidence of grade 1 complications could also be biased by the retrospective nature of the present study. Minor degrees of fever may have been overlooked and hence under reported. Furthermore, fever accompanied several other complications such as infections requiring additional antibiotics as well as a side effect of blood transfusion. But fever alone, responding well to antipyretics, was classified as grade 1 and observed in $3.1 \%$.

Our results showed that grade 2 complications were most common after PNL with a blood transfusion rate of approximately $8 \%$. This is much higher than that reported by El-Assmy et al. $[10,17]$ (approx 4\%) but comparable to those reported by Tefekli et al. (approx 10.9\%) [10]. The reason for higher rate of transfusion in our study group may be due to a substantial number of patients with complex calculi requiring multiple accesses. In our experience, performing additional punctures for residual stones and achieving a complete stone clearance, despite the increased risk of bleeding, was better accepted by our patients, than undergoing additional procedures for the same.

Renal hemorrhage requiring angioembolization was seen in only $4(0.5 \%)$ of our patients and owing to this small number we could not identify any risk factors that may have been responsible. El-Nahas et al. [18] in their retrospective study of 3,878 PNLs tried to identify various risk factors for hemorrhage requiring angioembolization. They found that upper calyceal puncture, solitary kidney, staghorn calculi, multiple site access and an inexperienced surgeon were significant risk factors. The incidence of extensive post-PNL hemorrhage was $1 \%$ in their study. Srivastava et al. [19] reported that approximately $1.4 \%$ of their patients required angiography or angioembolization for control of hemorrhage and found only stone size to be a significant risk factor.

Overall, grade 3a complications occurred in 6.4\% of our patients which is similar to those of Tefekli et al. [10] (6.6\%) but much higher than those reported by Moreno-Palacios et al. (4.5\%) [20]. About 3.5\% cases were of prolonged postoperative urinary leakage which were managed easily with DJ stenting. Munver et al. [21] reported intrathoracic complications with a rate of $16 \%$ for supracostal compared to $4.5 \%$ for subcostal tracts. In our study, intrathoracic complications requiring intercostal tube drainage occurred in $1.1 \%$ of cases.

Grade $3 b$ complications were seen in $3.5 \%$ of our subjects. However, some complications like urethral stricture and infundibular stenosis are seen long after the patient has been discharged. The Clavien-Dindo system was validated for a 30-day hospital stay and to include delayed complications into a system not validated for that length of time may actually introduce more heterogeneity. We believe that these complications are a result of unintentional intraoperative events and have therefore been included in our study.

Grade 4 complications consist of single or multi organ dysfunction requiring ICU care. These were seen in $1.2 \%$ of our cases. Four $(0.5 \%)$ of our cases suffered from acute renal failure in the immediate postoperative period. It is known that glomerular filtration rate decreases in the first 48 hours after PNL but acute renal loss or progression to end-stage renal disease is rare [22]. In fact, Bilen et al. [23] reported that almost all patients of chronic kidney disease treated with PNL had a net glomerular filtration rate benefit from surgery.

In the current study we also compared the complication rates between 2 groups of patients, one with simple and the other with complex calculi. We found that complications were significantly more common in patients harboring complex calculi compared to those presenting with simple calculi in Clavien groups 1, 2 and 3. Grade $4 \mathrm{~b}$ and 5 complications were exclusive to patients with complex calculi. Tefekli et al. [10] in their study found that grade 4 and 5 complications were seen with similar frequency in both the groups indicating that these complications may be a result of anatomic and technical problems compounded by underlying medical disorders.

We also performed a univariate analysis of the certain obvious factors that appear to have a bearing on the outcome of surgery in terms of the complication rates (table 4). In our study, we found that a positive preoperative 
urine culture and multiple accesses were associated with a significantly increased complication rate. Tefekli et al. [10] in their retrospective review concluded that diabetes mellitus and hypertension significantly increase the rate of complications after PNL. Moreno-Palacios et al. [20] in their study identified female gender, complex calculi and operative time $>120$ minutes as factors associated with severe complications. Our study is in concordance with various other studies which have found that the complications of PNL are statistically independent of body mass index [24-26].

The modified Clavien system classifies the complications in a standardized manner. It can also be used as a tool for internal quality assessment and evaluation of learning curves of a particular procedure [27]. However, the Clavien system does have some shortcomings. Grading of the same complication may differ across institutions. A procedure routinely done under general anesthesia at a certain institute may be done under local or regional anesthesia elsewhere. Hence, the patients grouped under category $3 \mathrm{a}$ at one institute may find themselves placed in category $3 b$ at another institute. The subdivision of group 3 based upon the type of anesthesia required and not the surgical procedure. Hence it fails to take into account the incidental morbidity of various ancillary procedures. For example, urethral stricture requiring optical internal urethrotomy may be sub classified in the same category as hemorrhage requiring nephrectomy. The indications for ICU care also differ among various centers. The rates of ICU admission are dictated by various factors like eco- nomic considerations of the patient, institutional policies, physician preference and insurance policy issues. ICU admissions may also be due to associated anesthetic complications and not surgical complications alone. Also, there is a lack of consensus on whether some events should be regarded as complications or routine sequelae of surgery. For example, peri-PNL leakage of urine in the early postoperative period has been considered a minor complication in some studies but not in others. There are certain complications like infundibular stenosis and urethral stricture which have a delayed presentation. Hence it is difficult to include these in a system which itself has been validated for only 30 days [6]. de la Rosette et al. [28] reported that the interobserver agreement in grading complications is modest. They found that interobserver reliability is low for minor complications but high for severe complications.

\section{Conclusion}

PNL is associated with a high degree of success and an acceptably low complication rate when used for the treatment of renal calculi. A simplified and well structured classification system for categorization of complications seems to be very attractive. The modified Clavien system, despite its various limitations, is a reasonable tool for grading and reporting of complications following PNL till a better system is proposed.

\section{References}

1 Fernström I, Johansson B: Percutaneous pyelolithotomy. A new extraction technique. Scand J Urol Nephrol 1976;10:257-259.

2 Preminger GM, Assimos DG, Lingeman JE, Nakada SY, Pearle MS, Wolf JS Jr: AUA guideline on management of staghorn calculi: diagnosis and treatment recommendations. J Urol 2005;173:1991-2000.

3 Matlaga BR, Kim SC, Lingeman JE: Improving outcomes of percutaneous nephrolithotomy. EAU Update Series 2005;3:37-43.

4 Michel MS, Trojan L, Rassweiler JJ: Complications in percutaneous nephrolithotomy. Eur Urol 2007;51:899-906.
5 Arslan MS, Zeytun H, Basuguy E, Arslan S, Aydogdu B, Okur MH: The outcome of percutaneous nephrolithotomy using intravenous catheter for obtaining percutaneous access as a treatment for renal stone disease in children: a pilot study. Urol J 2016;13:2502_ 2508.

6 Dindo D, Demartines N, Clavien PA: Classification of surgical complications: a new proposal with evaluation in a cohort of 6336 patients and results of a survey. Ann Surg 2004;240:205-213.

7 Gonzalgo ML, Pavlovich CP, Trock BJ, Link RE, Sullivan W, Su LM: Classification and trends of perioperative morbidities following laparoscopic radical prostatectomy. J Urol 2005;174:135-139.
8 Kocak B, Koffron AJ, Baker TB, Salvalaggio PR, Kaufman DB, Fryer JP, Abecassis MM, Stuart FP, Leventhal JR: Proposed classification of complications after live donor nephrectomy. Urology 2006;67:927-931.

9 De la Rosette JJ, Zuazu JR, Tsakiris P, Elsakka AM, Zudaire JJ, Laguna MP, de Reijke TM: Prognostic factors and percutaneous nephrolithotomy morbidity: a multivariate analysis of a contemporary series using the Clavien classification. J Urol 2008;180:2489-2493.

10 Tefekli A, Ali Karadag M, Tepeler K, Sari E, Berberoglu Y, Baykal M, Sarilar O, Muslumanoglu AY: Classification of percutaneous nephrolithotomy complications using the modified Clavien grading system: looking for a standard. Eur Urol 2008;53:184-190. 
11 Rassweiler JJ, Renner C, Eisenberger F: The management of complex renal stones. BJU Int 2000;86:919-928.

12 Muslumanoglu AY, Tefekli A, Karadag MA, Tok A, Sari E, Berberoglu Y: Impact of percutaneous access point number and location on complication and success rates in percutaneous nephrolithotomy. Urol Int 2006;77:340346.

13 Lee WJ, Smith AD, Cubelli V, Badlani GH, Lewin B, Vernace F, Cantos E: Complications in percutaneus nephrolitotomy. AJR Am J Roentgenol 1987;148:177-180.

14 Mousavi-Bahar SH, Mehrabi S, Moslemi MK: Percutaneous nephrolithotomy complications in 671 consecutive patients: a singlecenter experience. Urol J 2011;8:271-276.

15 Labate G, Modi P, Timoney A, Cormio L, Zhang X, Louie M, Grabe M, de la Rosette $\mathrm{J}$ : The percutaneous nephrolithotomy global study: classification of complications. J Endourol 2011;25:1275-1280.

16 Gutierrez J, Smith A, Geavlete P, Shah H, Kural AR, de Sio M, Amón Sesmero JH, Hoznek A, de la Rosette J: Urinary tract infections and post-operative fever in percutaneous nephrolithotomy. World J Urol 2013; 31:1135-1140.

17 El-Assmy AM, Shokeir AA, Mohsen T, ElTabey N, El-Nahas AR, Shoma AM, Eraky I, El-Kenawy MR, El-Kappany HA: Renal access by urologist or radiologist for percutaneous nephrolithotomy — is it still an issue? J Urol 2007;178:916-920.
18 El-Nahas AR, Shokeir AA, El-Assmy AM, Mohsen T, Shoma AM, Eraky I, El-Kenawy MR, El-Kappany HA: Post-percutaneous nephrolithotomy extensive hemorrhage: a study of risk factors. J Urol 2007;177:576-579.

19 Srivastava A, Singh KJ, Suri A, Dubey D, Kumar A, Kapoor R, Mandhani A, Jain S: Vascular complications after percutaneous nephrolithotomy: are there any predictive factors? Urology 2005;66:38-40.

20 Moreno-Palacios J, Maldonado-Alcaraz E, Montoya-Martínez G, Rivas-Ruiz R, Cedillo-López U, Okhunov Z, Serrano-Brambila EA: Prognostic factors of morbidity in patients undergoing percutaneous nephrolithotomy. J Endourol 2014;28:1078-1084.

21 Munver R, Delvecchio FC, Newman GE, Preminger GM: Critical analysis of supracostal access for percutaneous renal surgery. J Urol 2001;166:1242-1246.

22 Canes D, Hegarty NJ, Kamoi K, Haber GP, Berger A, Aron M, Desai MM: Functional outcomes following percutaneous surgery in the solitary kidney. J Urol 2009;181:154160.

23 Bilen CY, Inci K, Kocak B, Tan B, Sarikaya S, Sahin A: Impact of percutaneous nephrolithotomy on estimated glomerular filtration rate in patients with chronic kidney disease. J Endourol 2008;22:895-900.
24 El-Assmy AM, Shokeir AA, El-Nahas AR, Shoma AM, Eraky I, El-Kenawy MR, El-Kappany HA: Outcome of percutaneous nephrolithotomy: effect of body mass index. Eur Urol 2007;52:199-204.

25 Torrecilla Ortiz C, Meza Martinez AI, Vicens Morton AJ, Vila Reyes H, Colom Feixas S, Suarez Novo JF, Franco Miranda E: Obesity in percutaneous nephrolithotomy. Is body mass index really important? Urology 2014; 84:538-543.

26 Tefekli A, Kurtoglu H, Tepeler K, Karadag MA, Kandirali E, Sari E, Baykal M, Muslumanoglu AY: Does the metabolic syndrome or its components affect the outcome of percutaneous nephrolithotomy? J Endourol 2008;22:35-40.

27 Hruza M, Weiss HO, Pini G, Goezen AS Schulze M, Teber D, Rassweiler JJ: Complications in 2200 consecutive laparoscopic prostatectomies: standardized evaluation and analysis of learning curves. Eur Urol 2010; 58:733-741.

28 de la Rosette JJ, Opondo D, Daels FP, Giusti G, Serrano Á, Kandasami SV, Wolf JS Jr, Grabe M, Gravas S: Categorisation of complications and validation of the Clavien score for percutaneous nephrolithotomy. Eur Urol 2012;62:246-255. 